\title{
The Effect of Coloring on the Transmission of Electromagnetic Radiation in Eyeglass Lenses
}

\begin{abstract}
PAWEL PIETRUSIEWICZ*
Institute of Physics, Czestochowa University of Technology, Armii Krajowej 19 Av., $42-200$ Czestochowa, Poland

The paper presents the results of investigations on the transmission of electromagnetic radiation from the UV-Vis ranges of organic colored spectacle lenses. All investigated lenses had the same refractive index and one of them differed in the SPF (sun protection factor). The main criterion for the quality of colored eyeglass lenses is eye protection against harmful UV radiation, infrared radiation and excessive exposure to solar radiation. The lenses were measured using a Varian UV-Vis Cary model 300 spectrophotometer. These studies showed that the best protection against this radiation is the choice of coloring in a color other than blue, and that as the power of the colored lens increases, protection against harmful UV radiation decreases.
\end{abstract}

Keywords: colored lenses, anti-reflective coatings, light transmission

People have different senses: taste, smell, hearing and sight. If we think about it, without which of them would be the hardest to live for us, the answer is simple. It would be the most difficult for us to function without sight. People take care of themselves, lead a healthy lifestyle, use diets, creams with a filter on the skin, various types of cosmetics. They should also look after the eyes to the same extent because the eyesight is an organ that is not subject to regeneration itself. Most of the population wears corrective eyeglasses, more and more often reach for colored corrective glasses to improve the comfort of seeing, and thus, life. Companies producing corrective colored lenses have a full range of different colors, coatings and materials. Not all of us, however, focus on health benefits depending on our choice, buton aesthetic and fashion considerations. Most of us going on summer holidays, in addition to a bathing suit, bring various types of creams with a filter that protect our skin from the harmful effects of UV radiation. In recent years, we can also observe a greater social awareness related to the protection not only of the skin itself, but also of sight.

It should be emphasized that excessive exposure to UV radiation has an adverse effect not only on our skin, accelerating the aging process, but also on the state of our sight. In extreme cases, the long-term lack of protection against harmful UVA and UVB radiation can lead to pathological changes of the eye and even blindness. The eye has the ability to absorb UVA and UVB radiation. Both the cornea and the lens absorb the largest amount of sunlight. Radiation with a wavelength below $300 \mathrm{~nm}$ (UVB) is primarily absorbed by the cornea. The lens absorbs mainly UVA radiation (wavelength less than $370 \mathrm{~nm}$ ) [1]. In contrast, radiation from the $380-400 \mathrm{~nm}$ range reaches the retina [2,3]. UV radiation very easily damages the conjunctiva, which activates a series of complex oxidative reactions leading to cell death. Eye tumors often develop on the conjunctiva near the hem of the cornea. Studies have shown that malignant melanoma and choroidal melanoma, occur 10 times more often in the race with light skin than in people with black race. Strong exposure of UV radiation often leads to the development of the wings [4]. In recent years, the diversity of consumer needs has led to the introduction to the optical market of a huge number of different products, tailored to individual professional and hobby preferences. Current glasses are designed to increase comfort and safety. Today's optical salons offer a wide range of glasses enriched with different coatings [5-17]. It is also worth mentioning the development of optics in the use of lenses for scientific purposes - such as the construction of microscopes. Further development of the optics allowed to get to know what can not be seen with the human eye, even with a fully healthy eye, without the use of glass. Notall glasses provide adequate protection against UV radiation. Therefore, the glass may have an additional function, namely protection from strong sunlight. For this purpose, two versions of lenses can be used: photochromes and permanently fixed lenses. Lenses that are colored are often considered as refined glass. In optical salons there is a huge range of dyes possible to be made on organic lenses. Each color is possible. The situation is worse in the case of mineral glasses. Only brown and gray colors are available here. Lenses can be colored entirely in one or two colors and graded. When choosing the strength of color, the type of use of the glasses, degree of insolation and individual sensitivity to light should be taken into account.

The work aims to show the effect of coloring corrective lenses on the transmission of electromagnetic radiation in individual, stained corrective lenses, supported by studies conducted on the UV-Vis photosphere.

\section{Experimental part \\ Methodology}

The research involved 9 color correction lenses with the same refractive index - 1.50, but with different powers, staining and other SPF (sun protection factor). Studies on the color properties of spectacle lenses were carried out at the Institute of Physics at the Czestochowa University of Technology using a Varian UV spectrometer - UV-Vis model 300. The material was studied in the field of electromagnetic waves with a length of $200 \mathrm{~nm}-900 \mathrm{~nm}$. This spectrophotometer is a double beam instrument controlled by a computer with a monochromator and a premonochromator. Lenses were divided into three groups.

\footnotetext{
* email: pietrusiewicz.pawel@wip.pcz.pl
} 


\begin{tabular}{|l|c|c|c|c|}
\hline Label & Color & Power & Coeficient SPF & Additional layers \\
\hline \hline S1 & gray & -0.25 & 25 & Gold mirrored \\
\hline S2 & gray-green & -0.25 & 25 & Silver mirrored \\
\hline S3 & blue & -0.25 & 25 & Orange mirrored \\
\hline S4 & gray-green & -0.25 & 25 & polarization \\
\hline S5 & inky & -0.25 & $\mathbf{2 5}$ & polarization \\
\hline S6 & gray & -2.75 & 25 & - \\
\hline S7 & brown & -2.75 & 25 & - \\
\hline S5 & gray & -0.25 & 25 & - \\
\hline S9 & bigradal mix of & -0.25 & $\mathbf{5 0}$ & - \\
& colors & & & \\
\hline
\end{tabular}

Table 1

LIST OF PARAMETERS OF THE TESTED LENSES
The first group is a lens with a power of -0.25 with a graygreen $85 \%$ color with additional mirrored coatings in different colors. The second group is a lens with polarization, staining 85\% in two different shades - gray green and ink. The third group are lenses with standard $85 \%$ staining in brown and gray colors. For comparison, the lens was also measured - 0.25 with an $85 \%$ staining, which differs from the rest of the SPF. In the case of the latter, it is SPF - 50, while in the remaining SPF - 25. The exact characteristics of individual subjects are presented in table 1.

\section{Results and discussions}

The first group consisted of three lenses with the same breaking power, ie - $0.25 \mathrm{dph}$ and with the same SPF - 25 $(S 1, S 2, S 3)$. Each of these lenses is made of the same material with an index of 1.50. How ever, they differ in the color of the staining and the color of the mirror coating applied to the outer surface of the lens. Thus: S1 has gray dyeing and a mirror coating in a golden color, S2 has graygreen dyeing and a silver mirror coating, S3 has blue dyeing and an orange mirror coating. The dependence of transmittance on individual wavelengths for the lenses in this group is shown in figure 1.

Light spectra for S1 and S2 are arranged in similar curves, which we can not say about the spectrum for S3, which differs significantly from the first two. Two significant decreases were observed with the S1 lens at a wavelength of $447 \mathrm{~nm}$, i.e. for the blue color and $574 \mathrm{~nm}$, i.e. for the green color. The plot of the S2 lens shows a significant decrease in the light spectrum permeability for a wavelength of $585 \mathrm{~nm}$, i.e. for the orange-yellow color. For the S3 lens, a significant decrease in the lighttransmission permeability is observed for a wavelength of approx. 537 $\mathrm{nm}$, i.e. for the green color. It is worth emphasizing that lenses with a mirror coating are the only ones that exhibit low red color transmittance, which does not have a positive effect on our eyes. It follows that the mirror coating performs not only an aesthetic function, but above all a protective function. By entering the research, it was thought that the blue lens would absorb this color best. Research shows that it is exactly the opposite. The staining color results in greater permeability of waves of the same color. The blue color transmits blue radiation, which is unhealthy for our eyes, so better choose lenses in a different color than this one.

The second group of lenses was composed of two polarized lenses with the same power, ie - $0.25 \mathrm{dph}$ and with the same SPF - $25(\mathrm{~S} 4, \mathrm{~S} 5)$. Each of these lenses is made of the same material with an index of 1.50. However, they differ in the color of the dyeing. Thus: S4 has a graygreen color, while S5 has an ink-dyeing. The dependence of transmittance on individual wavelengths for lenses from this group is shown in figure 2.

Light spectra for S4 (fig. 2a) and S5 (fig. 2b) are arranged in similar curves only up to approx. $450 \mathrm{~nm}$. In the case of the $\mathrm{S} 5$ lens (fig. 2b), the absorption of light falls in the range from about $500 \mathrm{~nm}$ to $420 \mathrm{~nm}$. At the S4 lens, three significant decreases were observed for the wavelength: $446 \mathrm{~nm}$, i.e. for the blue color, $548 \mathrm{~nm}$, i.e. for the green color, $715 \mathrm{~nm}$, i.e. for the red color. The 55 lens plot shows high low transmittance for wavelengths of $586 \mathrm{~nm}, 629$ $\mathrm{nm}$ and $776 \mathrm{~nm}$. that is for orange and red. It should be emphasized the difference between these two lenses, that the 54 lens separates very little from blue to indigo, while both are characterized by high red and orange absorption,
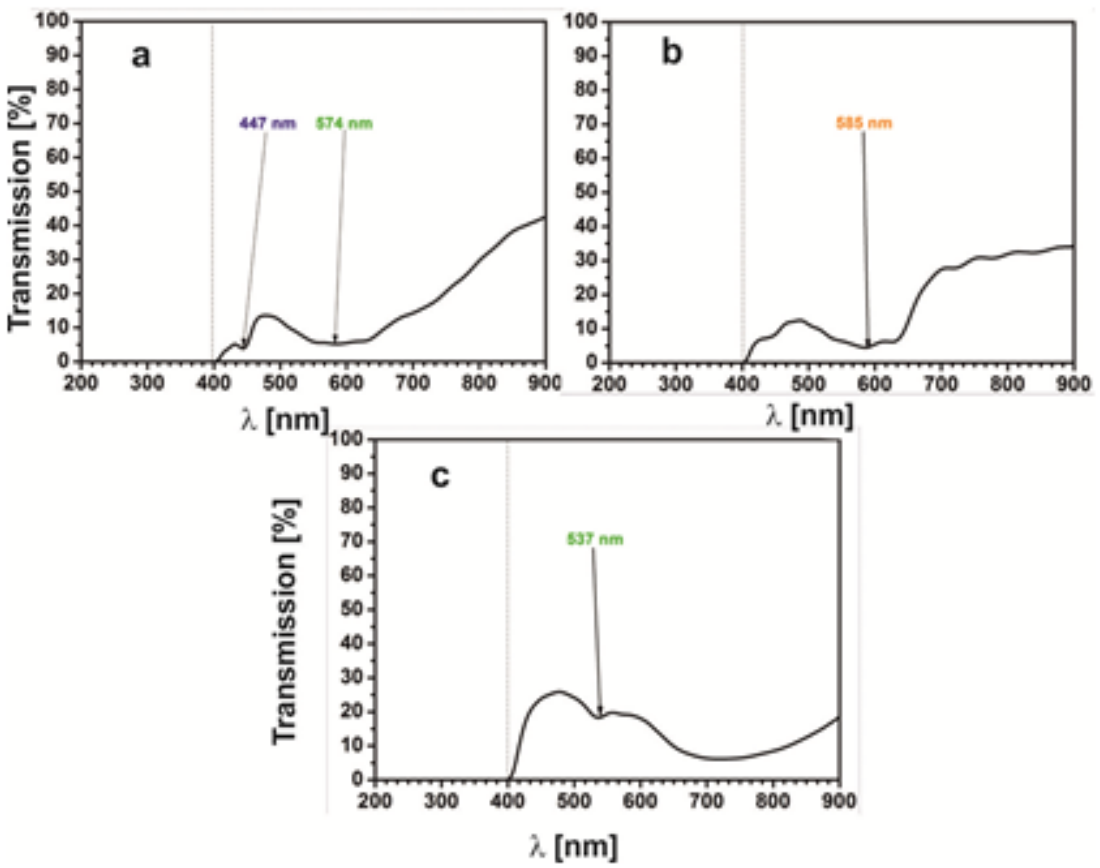

Fig. 1. The dependence of electromagnetic radiation transmission on the wavelength of a) S1, b) S2, c) S3 

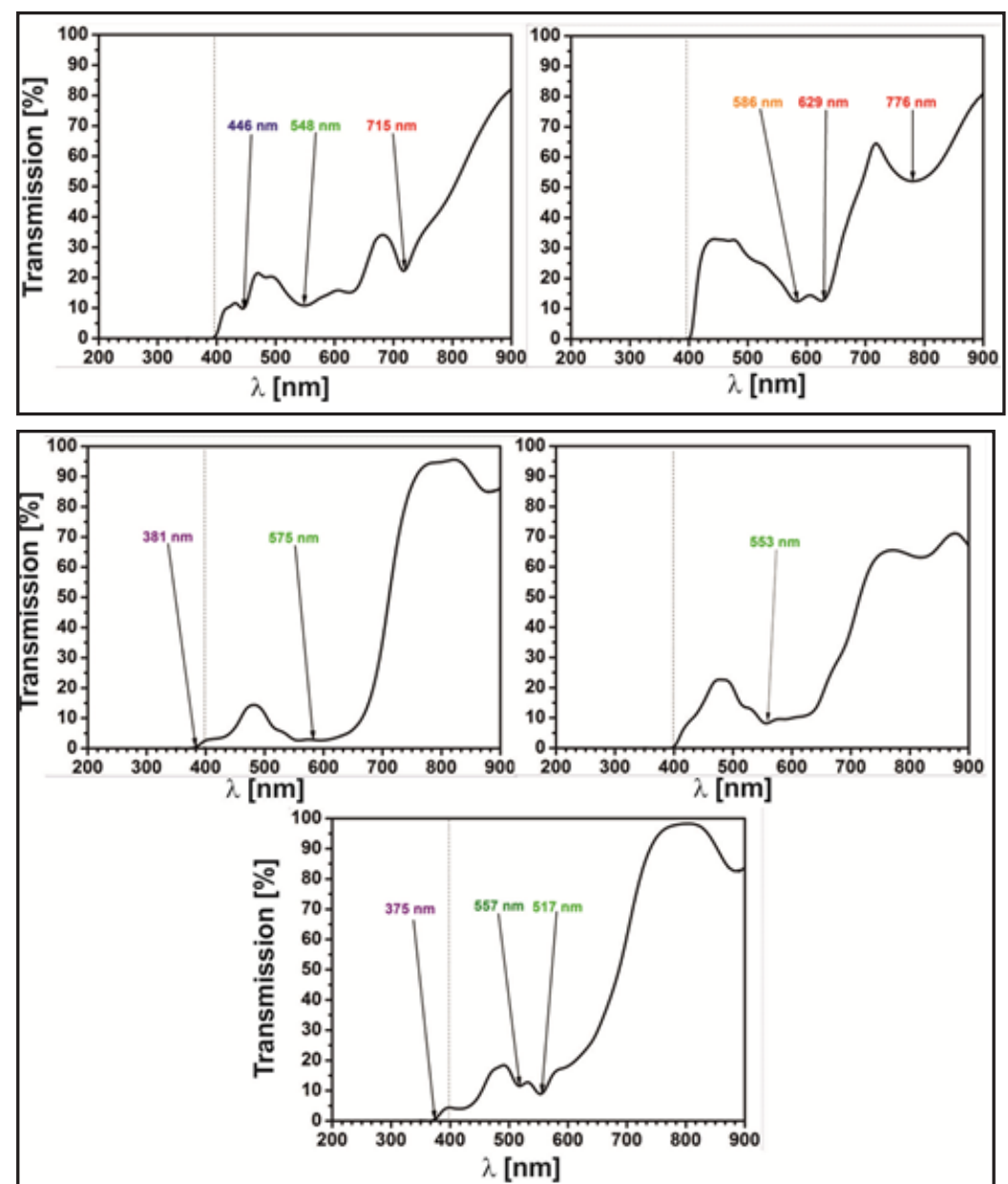

which are unhealthy for our eyes. S4 lens. to a significant extent cuts off the blue color, which indicates that graygreen staining positively affects the protection of vision. S5 lens. shows a large transmittance unhealthy for the eyes, blue. This is due to the dyeing color, which in this case is ink-jet, i.e. dark blue.

In the third group, three lenses with different breaking strength and the same SPF - 25 (S6, S7, S8) were used for the measurements. Each of these lenses is made of the same material with an index of 1.50 . S6 is a lens with a breaking power - $2.75 \mathrm{dph}$, dyed gray in 85 percent. S8 is a lens with identical parameters, but with a power of 0.25 dph. 57 is a lens with a power $-2.75 \mathrm{dph}$, colored 85 percent brown. The dependence of transmittance on individual wavelengths for the lenses in this group is shown in figure 3.

Light spectra for S6 (fig. 3a), S8 (fig. 3b) and S7 (fig. 3c) are arranged in similar, almost identical curves. However, the 56 lens has a much higher spectral absorption than in the $\mathrm{S} 8$ lens at a wavelength from $550 \mathrm{~nm}$ to $450 \mathrm{~nm}$ (from green to blue). In S6, significant decreases in absorption were observed at $381 \mathrm{~nm}$, i.e. for violet and $575 \mathrm{~nm}$, i.e. for green. In S8, we observe a decrease in transmittance at $553 \mathrm{~nm}$, i.e. with the green color. The S7 lens graph shows significant decreases in light spectrum permeability for wavelengths: $375 \mathrm{~nm}, 557 \mathrm{~nm}, 517 \mathrm{~nm}$, i.e. successively for violet, dark green and light green. It is worth paying special attention to $\mathrm{S} 6$ and S7 here. Both lenses with a breaking power - $2.75 \mathrm{dph}$, despite the different color of the color, do notequally cut off the color of purple, ie harmful to the eyes UVA radiation, to the same degree. S8 lens with breaking power - 0.25 dph shows UVA transmittance at zero level. The charts also show ed that the S7 lens and the S8 lens do not absorb blue and red, which have a negative effect on the health of our eyes.
Fig. 2. The dependence of electromagnetic radiation transmission on wavelengths for a) S4, b) S5

Fig. 3. The dependence of electromagnetic radiation transmission on the wavelength for a) S6, b) S7, c) S8.
The last $\mathrm{S9}$ lens was examined to determine the effect of the SPF on the transmittance of individual wavelengths. The study measured the lens with breaking power -0.25 $\mathrm{dph}, \mathrm{SPF}$ - 50 coefficient, colored in a way where colors of various intensities mix - violet, red, blue. The dependence of transmission on individual wavelengths is shown in figure S9 (fig. 4).

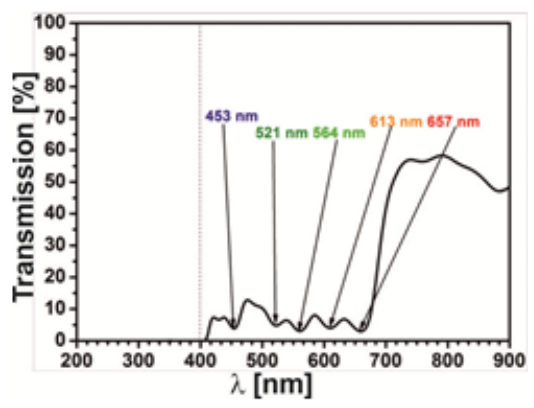

Fig. 4. The dependence of electromagnetic radiation transmission on the wavelength of $\mathrm{S9}$ lenses

The light spectrum for $\mathrm{S9}$ (fig. 4) is arranged in a surprising way. The most significant decreases in transmittance were observed among all the lenses that are located at the wavelengths: $453 \mathrm{~nm}, 521 \mathrm{~nm}, 564 \mathrm{~nm}$, $613 \mathrm{~nm}, 657 \mathrm{~nm}$, i.e. successively the colors: blue, dark green, light green, orange and red. S9 stands out from the other lenses with the smallest transmittance for each wavelength, which means that it is healthy for our eyes.

\section{Conclusions}

The study used 9 corrective lenses of different strengths, staining and colors of these stains. In the first group, where the lenses differing only in the color of the color and the color of the mirror coating, the influence of the color of the lens on the transmittance of the light beam is evident. A gray and gray-green lens cuts the beam almost to the same degree. The blue lens, on the other hand, has less absorption 
for 450 - 500 waves, that is from indigo to blue. This indicates that there is a direct dependence of the absorption of a given color on the color of the lens. It is worth noting that these three lenses are generally characterized by low transmittance, which determines the mirror coatings. In summary, the lens of the blue color, will let more light into the eye with the same color, and by applying an additional coating in the form of a mirror, we increase the absorption of visible light. In the second group, two lenses of identical properties were measured, butother dyeing, i.e. gray-green and ink-colored. The ink color is like a mixture of two basic colors - purple and indigo. The graygreen color is more saturated with green. Here, too, we clearly see the dependence of the dyeing of the lens and the wave of the same color. The curve of the S4 (graygreen) lens jumps, which is evidence of the use of different colors during the dyeing process. The inkjet lens absorbs much of the colors close to its own to a lesser extent. In the third group, two polished gray-stained polarized lenses were investigated, including one with a power of $0.25 \mathrm{dph}$, the other with $2.75 \mathrm{dph}$ and one brown with a power of $2.75 \mathrm{dph}$. Studies have shown that also in this case the light spectrum runs similarly for lenses of the same color. However, the $2.75 \mathrm{dph}$ lens does not protect our eyes from harmful UV radiation, which means that the higher the power of the lens towards the minus, the more our eyes are more exposed to the harmful effects of light. Polarization does not affect transmittance. For comparison and subsequent analysis, a multi-colored lens was measured with twice the SPF, which has a significant influence on the transmittance of colored spectacle lenses. In addition, the thesis on the influence of dyeing color on absorption is again confirmed. The $\mathrm{S9}$ lens is specific. It is a mixture of different staining, which go smoothly into one another, which can be seen in the curve of light beam transmission and what again proves the huge effect of color staining on our comfort of looking through colored corrective glasses.

To sum up, the research has shown that the level of electromagnetic radiation transmittance is mainly influenced by: Color of the lens; Power breaking the lens and Coatings applied to the outer surface of the lens - SLR.

Colored lenses are the most safe for our eyes, but when you order glasses, you can consciously increase the level of eye protection by choosing the right coatings, ie SLRs.
There is much talk about eye radiation harmful to the eyes. Studies have shown that the best protection against this radiation is the choice of coloring in a different color than blue. The results showed that as the power of the colored lens increases, protection against harmful UV radiation decreases. For such patients, to fully protect their eyesight, it is recommended to wear corrective contact lenses and sunglasses without correction, preferably those with a mirror coating.

\section{References}

1.GILLAN, J.G, Can J Ophthalmol., 5, 1970, p.146

2.WERNER, J.S., J. Opt. Soc. Am., 72, 1982, p. 247

3.WEALE, R.A., J. Phys., 395, 1988, p. 577

4.HIRST, L., Pterygium. ed H. TAYLOR (The Hague, The Netherlands: Kugler Publications) 2000, p. 15-27.

5.DU, Y., LUNA, L.E., TAN, W.S., RUBNER, M.F., COHEN, R.E., ACS Nano., 4, no. 7, 2010, p. 4308

6.MINOT, M.J., J. Opt. Soc. Am., 66, 1976, p. 515

7.CHEN, C.C., LIN, D.J ., DON, T.M., HUANG, F.H., CHENG, L.P., J. NonCrystalline Solids., 354, 2008, p. 3828

8.XU, Q., LIANG, L., HU, M., SUN, H., ZHOU, L., XIANG, G., Guangxue Xuebao/Acta Optica Sinica, 32, 2012, p. 1216001

9.BOLTAU, M., WALHEIM, S., MLYNEK, J., KRAUSCH, G., STEINER, U., Nature, 391, 1998, p. 877

10.WALHEIM, S., SCHÄFFER, E., MLYNEK, J., STEINER, U., Science, 283, 1999, p. 520

11.GARUS, S., GARUS, J., SZOTA, M., NABIAfEK, M., GRUSZKA, K.,

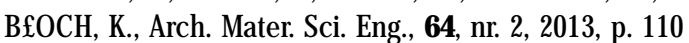

12.SANDU, A.V., BEJ INARIU, C., NEMTOI, G., SANDU, I.G., VIZUREANU, P., IONITA, I., BACIU, C., Rev.Chim. (Bucharest), 64, no. 8, 2013, p. 825.

13.IOANNOU, P.D., NICA, P., PAUN, V., VIZUREANU, P., AGOP, M., Physica Scripta, 78, no. 6, 2008, Article Number: 065101

14. VIZUREANU, P., Environmental Engineering And Management Journal, 8, no. 2, 2009, p. 301

15.VIZUREANU, P., PERJU, M.C., GALUSCA, D.G., NEJ NERU, C., AGOP, M., Metalurgia International, vol. XV, no. 12, 2010, p. 59-64.

16.NEJ NERU, C., PERJ U, M.C., SANDU, A.V., AXINTE, M., QUARANTA, M., SANDU, I., COSTEA, M., ABDULLAH, M.M.A.B., Rev. Chim. (Bucharest), 67, no. 6, 2016, p. 1191-1194.

17.LUPU, D., PETRISOR, M.B., BERCU, A., TOFAN, M., Emerging Markets Finance And Trade, 54, no. 3, 2019, p. 552.

Manuscript received: 9.09 .2018 